\title{
Policy Based Handover In Wireless and Mobile Networks
}

\author{
Hakima CHAOUCHI \\ CTR, King's College, University of London \\ Strand London WC2R $2 L S$ \\ United Kingdom
}

\begin{abstract}
Wireless networks bring a new market to service providers and terminal manufacturers. In fact after the success of the wired internet, wireless and mobile internet arrives with the ambition of providing new services to Internet users. However wireless networks face important challenging issues before attaining a similar or better success than the wired Internet. These issues are mainly related to the classical QoS, mobility, security problems, and the wireless specific problems such as the radio resource characteristics. In this article we are interested by the mobility problem and particularly the handover management problem. We introduce a policy based handover and we propose to combine the mobility management with the policy based architecture in order to achieve policy based handovers that are triggered not only on the signal strength basis, but also on policy constraints such as cost, resource availability or load balancing. The policy based architecture will provide the handover triggering mechanism and the decision support related to the best next cell where the mobile node will move. We also introduce mobility parameters in the Service Level Specification (SLS) that specifies the handover type, the accepted handover packet loss, and the accepted handover delay. Based on these SLS mobility parameters, the QoS service classes can be mapped to the smooth (minimum packet loss), the fast handover (minimum delay), or the seamless handover (fast and smooth).
\end{abstract}

Key words: Micro mobility, fast handover, smooth handover, seamless handover, context transfer, .Mobile IP, Mobile IPv6 fast handover, policy based management.

The original version of this chapter was revised: The copyright line was incorrect. This has been corrected. The Erratum to this chapter is available at DOI: 10.1007/978-0-387-35703-4_21 


\section{INTRODUCTION}

In order to avoid the service disruption, the handover management framework must perform a smooth and/or fast handover. A smooth handover requires minimum packet loss and a fast handover requires minimum delay. The handover is composed of an initiation phase, a decision phase and an execution phase. During the initiation phase, the mobile node detects the neighbouring cells. During the decision phase, the mobile node decides to switch to the next cell. Finally, during the execution phase, the mobile node configures its wireless interface to move to the next cell.

Several mobility schemes and particularly Mobile IP scheme have an important drawback which is to perform all the actions related to the mobility of the node after the handover execution phase. That means the physical layer is connected to the physical layer of the new cell, but the network layer is not already configured to use the resources of the new cell. In Mobile IP, the mobility actions needed to be performed after the handover execution are mainly the authentication, getting the new IP address and the new location registration of the mobile node. Why not perform certain mobility functions during the handover initiation in order to minimize the delay due to all these mobility actions?

Mobile IPv6 fast handover introduces the predictive handover concept which is triggered by the fading of the signal strength in the current cell. In addition, Fast Mobile IP assumes to have the information related to the next cell and proposes to establish a tunnel between the current access router and the next access router in order to forward the mobile node packets during the handover. However, the information related to the next cell is not always available.

In this paper we introduce in a given mobility management architecture a policy mobility manager which will trigger a predictive handover based on constraints other than the fading of signal strength such as the cost or the resource availability in the neighbouring cells. The policy mobility manager will also provide the next cell selection decision. In addition, it will provide the information related to the selected next cell to the current access router in order to facilitate the definition of a new care of address and establish the tunnel to the next access router and forward the mobile node packets during the handover. The predictive handover will achieve a fast handover and the mobile node packet forwarding will achieve the smooth handover.

The policy mobility manager is the Policy Decision Point (PDP) in the IETF policy based management architecture [1] illustrated on Figure 1. 


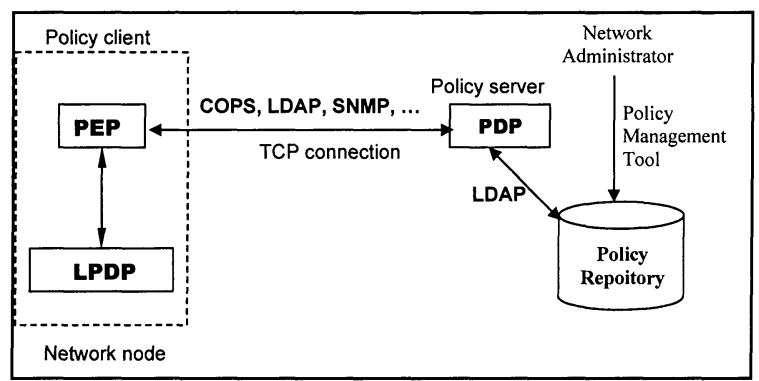

Figure 1. IETF Policy Based Management architecture.

Policy based management is an interesting approach to facilitate the automatic configuration of the network elements by using the policy concept [1]. The policy is a rule composed by a condition part and an action part which is triggered when the condition part is satisfied. Policy management tool allows the translation of high level policies to low level policies in order to configure the network elements or to achieve other actions in the network [1]. In the case of QoS management, many efforts have been spent at the IETF to achieve the configuration of the network elements. For this purpose, the IETF defines the policy based architecture and introduces a policy transport protocol named COPS (Common Open Policy Service) [2] used between the policy server (Policy Decision Point-PDP) and the network elements' policy enforcement points (PEP) in order to achieve the automatic configuration of the network elements [3]. LPDP is the Local Policy Decision Point.

The rest of this paper is structured as follow. First, we present an overview on the handover management schemes. Then we propose our policy based handover architecture combined with a mobility management, and the protocol communication. We also introduce the mobility parameters in a Service Level Specification and the possible mapping of the QoS service classes to the corresponding handover type. Finally, we present our tested and future work and we conclude this work.

\section{HANDOVER MANAGEMENT OVERVIEW}

Mobile IP [4] is the widely known proposal within IP mobility management. It is mainly based on the usage of two addresses for the mobile node. The MN gets a home address from its home network, it's a permanent address. Each time the mobile node connects to a foreign network it will get a temporary address which is its care of address. In order to keep track of the 
mobile node, it performs a registration of its new location at the home network. The home network sends the packet to the mobile node by using this registered location. Mobile IP suffers from several weaknesses that have led to the definition of the macro/micro-mobility architecture.

HMIPv6 (Hierarchical Mobile IPv6) [5] and Mobile IPv4 Regional registration [6] are enhancements schemes of Mobile IP, they propose to minimize the delay due to the registration phase of the mobile node by using a hierarchical or a regional registration, they intend to support the micro mobility management. They propose to make the registration of the mobile node locally by an anchor or regional mobility agent present in each visited domain.

Cellular IP [7] and Hawaii [5] bring a new approach for the management of the micro-mobility. Specialized mobility in Cellular IP acts as a gateway towards the internet as a Mobile IP FA. Cellular IP aims to replace IP in the wireless network. Cellular IP routing is based on routes established and updated by the MN during the connection to the network. All these routes bind a mobile connected to the network and the gateway. Each station maintains a routing cache table that allows it to forward packets from the gateway to the $\mathrm{MN}$, or vice versa. The routes are established and maintained by a transmission of special control packets that trigger the stations on the path to update their routing cache. A beacon is periodically sent by the gateway to flood the network. The MN sends route update when it connects to the network and each time it changes its point of attachment. These packets sent hop by hop towards the gateway trigger the stations to update their routing cache. There are two handovers in cellular IP; the hard handoff and the semi-soft handoff. The hard handoff provides no guarantee while the semi-soft handoff provides a minimum packet loss. Cellular IP present a native support for passive connectivity with a paging mechanism. In fact, some stations maintain paging caches that are used in case of paging request.

Hawaii [5] works above IP. Each Hawaii station in the network must act as an IP router with a specific Hawaii feature. Each station maintains a routing cache to manage the mobility. The hop-by-hop transmission of special packets in the network triggers the stations to maintain their routing caches. The network is organised as a tree with a gateway as the root of this tree. Hawaii defines two different handovers adapted to different radio access technologies. It supports the passive connectivity with a paging mechanism. The geographic paging areas are composed of stations belonging to the same IP multicast group. The classical multicast mechanism is used to send the paging requests.

Fast handover MIPv6 [8] proposes to provide a minimum handover delay by using a predictive handover or a layer two triggered handover. The handover is generally triggered due to the fading signal strength. However, 
the handover may be triggered by other constraints such as cost or available resources. Especially in a heterogeneous $4 \mathrm{G}$ wireless network where the cost and the available resources can differ from one technology to another in the same mobile node coverage area.

\section{POLICY BASED HANDOVER MANAGEMENT}

\subsection{The proposed architecture}

Most of the mobility management schemes lack the support of handovers triggered by other constraints than the fading strength of the signal. We define a policy based handover as a handover triggered not only by the fading of the signal strength but on other constraints such as cost or resource availability or the provider policy. For instance the network provider may decide to change the point of attachment of certain mobile nodes for load balancing or for better QoS. Policy based handover will be very useful in a $4 \mathrm{G}$ wireless and mobile networks where a mobile node can choose between different access networks technologies the best one to support his service [9].

We propose a policy based management to support the triggering of the policy based handover and the policy based decision mechanism selecting the next cell where the mobile node will move to. In addition we propose the new mobility parameters in the Service Level Specification which are used to select the handover corresponding to the mobile node service class.

Our architecture considers' a set of wireless access networks. Each wireless access network is connected to the wired network by border routers (BR) which connects a set of base stations or access routers (AS) wireless cells to the wired access network. The wired access network connects the wireless access networks to the core network. The authentication, authorization, accounting (AAA) server in the wired network is used to achieve the authentication of the mobile users; it is used in interaction with the mobility protocol [10]. We assume that there is already a mobility management protocol deployed between the mobile nodes and the access routers. In this paper we consider as an example Mobile IPv6 and its fast handover scheme which provide a support for a predictive handover.

The proposed policy based architecture illustrated on Figure 2 is composed by a Policy Mobility Manager (PMM) in each domain. Under the same administrative policy, a domain is composed by a wired access network connecting a set of wireless access networks to the core network. On Figure 2, we illustrate a Home and Foreign networks or domains. A 
Policy Mobility Client (PMC) is deployed on mobile nodes or access or border routers. The Policy mobility client (PMC) will communicate with the Policy mobility manager in order to achieve the policy based handover The policy Mobility Manager is responsible of triggering the policy based handover and selecting the best next cell to the mobile node as well.

It is interesting to make the Policy Mobility Manager decide the new location of the mobile node that can be used to consider different constraints during the decision process and enable QoS aware handover or to support vertical handovers or any constraint based handover. Using this policy based handover architecture; we can achieve micro, macro, intra and inter domain horizontal and vertical handovers.

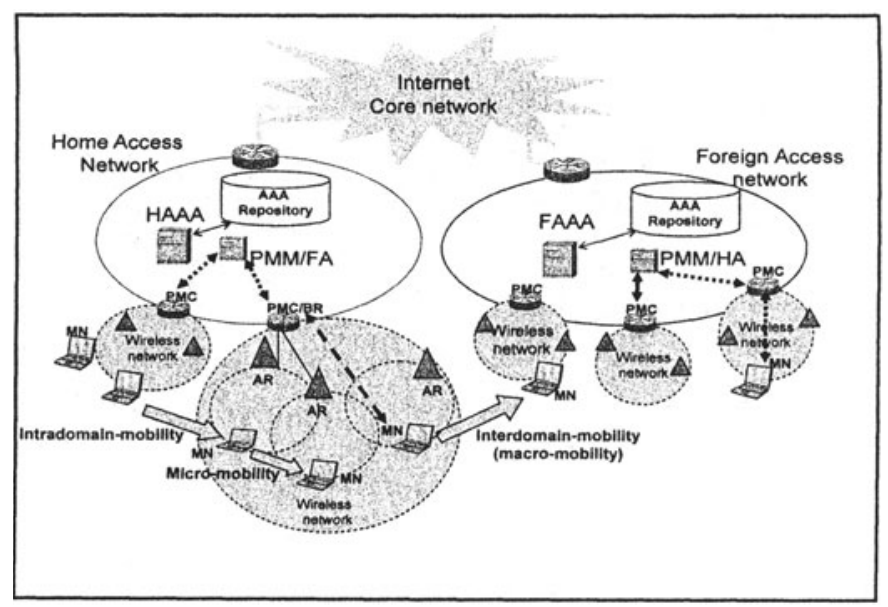

Figure 2. Policy based handover management architecture.

In the handover procedure, the mobile node may be able to be connected to two cells simultaneously. When using MIPv6 fast handover scheme, it can achieve a predictive handover based on the layer two triggers. If the mobile node cannot be connected to two cells simultaneously, MIPv6 fast handover scheme propose to achieve a predictive handover at the network layer, but it assumes there is another mechanism which provides the information about the next cell. Precisely, our policy based architecture will provide this information and make the predictive handover happen. In addition, the policy mobility manager will provide the information related to the selected next cell in order to facilitate to the current access router provide a new care of address and also construct a tunnel to the selected access router to forward packets or transfer the context of the mobile node.

Since the mobile node can/cannot support to be connected to two wireless cells simultaneously, we consider two scenarios. 
Scenario 1: the mobile node can be connected to two access routers simultaneously

As illustrated on Figure 3, the mobile node in his current location sends to the current access router the identity of the detected new access routers from which the mobile node receives an advertisement during the handover initiation phase. This information is forwarded by the Policy Mobility client at the access router to the Policy mobility manager that uses it to identify which are the possible cells where the mobile node may move to. The mobility manager will decide based on different constraints (QoS, domain policies ...) which is the suitable cell and replies to the mobility client of the mobile node to configure its wireless interface to move to the selected cell. In this scenario, the Policy mobility manager can also trigger the handover if necessary.

Scenario 2: the mobile node cannot be connected to two access routers simultaneously

If the mobile node cannot receive the new cell advertisement without breaking the current connection, the policy mobility manager can use topology information and the current location of the mobile node to identify the neighbour cell that can support the incoming mobile node. As illustrated on Figure 4, the policy mobility manager will request the policy mobility client to trigger the handover which will be achieved by the mobility management protocol.

\subsection{Protocol communication}

Depending on the scenario 1 or 2, the initiation handover message can be sent by the mobile node or by the policy mobility manager. Figure 3 and 4 illustrate the interaction between the mobile node (MN) and the network elements (cAR-current Access Router, PMC-Policy Mobility Client, BRBorder Router, PMM-Policy Mobility Manager, nAR-new Access Router) to achieve the policy based handover in scenario 1 and 2 respectively.

In Figure 3, the mobile node (MN) sends a handover solicitation message to the current access router. The PMC on the access router interacts with the PMM in order to better select the next cell to the mobile node. The PMM replies with the information related to the selected next cell. The cAR replies to the $\mathrm{MN}$ which executes its handover to the selected cell. If the mobility management protocol doesn't support the packet forwarding to the next cell, the PMM requests the PMC to configure the cAR to forward the packets to the selected nAR using the corresponding configuration policies. This request can be included in the next cell selection decision message. 


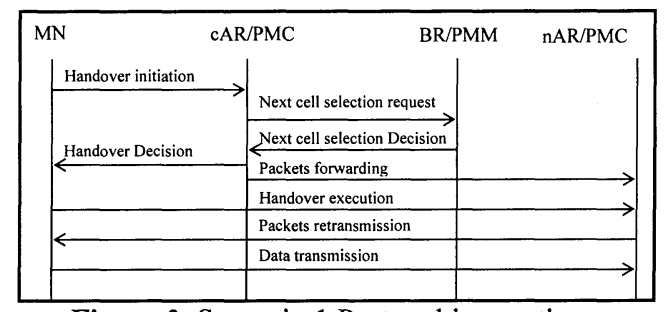

Figure 3: Scenario 1 Protocol interaction

Figure 4 shows the scenario 2, here the PMM initiates the handover and then the mobility management will handle it. The PMM will also request the cAR to forward the packets to the nAR.

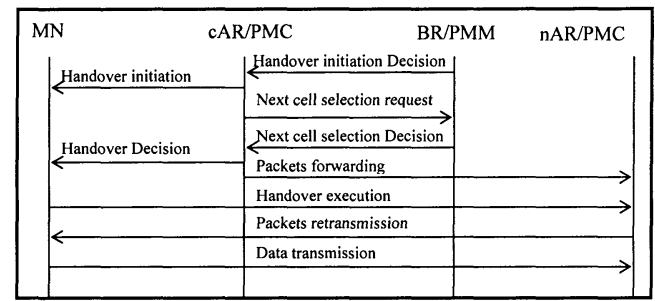

Figure 4: Scenario 2 Protocol interaction

Note that the policy based management architecture provides the automatic configuration using the PDP (here a PMM) decisions policies enforced by the PEP (here a PMC).

\subsection{Extended policy based handover management to the mobile node}

We can extend our policy based management to the mobile node by implementing the Policy mobility client in the mobile node. In this case, we can either totally integrate the mobility management protocol with the policy transport protocol, or just make them interact at the mobile node and use separate protocols to the mobility management and the policy based handover triggering and policy based next cell selection and packet forwarding and context transfer configuration.

\subsubsection{Separated mechanism}

In the separated mechanism approach, as illustrated on Figure 5 the PMM sends a handover initiation to the PMC at the MN. Then, the MN achieves the handover using the mobility management protocol. The PMM will provide the policy based selection of the next cell to the mobile node. It will 
also provide support for the forwarding of the packets from the cAR to the nAR and context transfer as well.

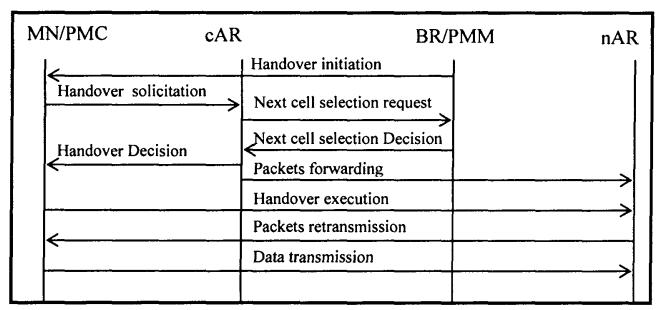

Figure 5 : Separated approach

\subsubsection{Integrated approach}

In the integrated scenario, as illustrated on Figure 6, the PMM sends a handover initiation to the PMC which will achieve the handover by configuring the mobile node to switch to the next nAR. The PMC will use the policy transfer protocol to exchange the mobility information.

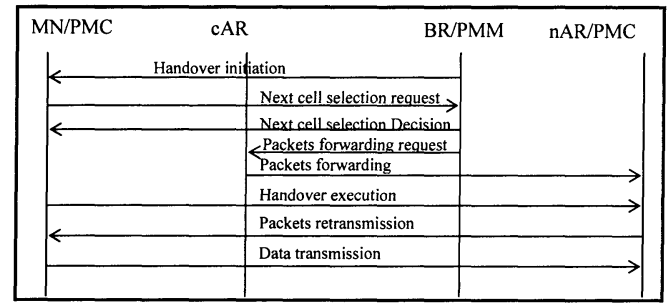

Figure 6 : Integrated approach.

The PMM will provide the policy based selection of the next cell to the mobile node. It will also provide support for the forwarding of the packets from the $\mathrm{cAR}$ to the $\mathrm{nAR}$ and context transfer configuration.

\subsubsection{Interaction with Location management}

If the mobile node can not provide the identity of the new cells, the policy based management can interact with a location management system in order to find the current location of the mobile node and define the possible next cells to the mobile node. In fact, Global positioning system can be used in interaction with the policy based architecture in outdoor environment to provide the current location of the mobile node. In an indoor environment, the policy based management mechanism can be used to provide location management. In fact policy based management protocol is a statefull protocol, it uniquely identify the policy clients (PMC). In both indoor and 
outdoor environment, the policy mobility manager needs network topology information in order to select the next cell to the mobile node using its current location.

\subsubsection{Interaction with AAA management}

In order to better minimise the delay due to the authentication procedure, we propose that the policy mobility manager during the predictive handover interacts with the AAA server in order to get a session key to the mobile node. This key will be delivered to the mobile node that will use it when he is connected to the selected new cell. The new access router authenticates the mobile node with this key locally. This key can also be used to identify the service characteristics that will be delivered to the mobile node. The mobility manager can request to the selected cell access router to establish the required characteristics of the service that will be provided to the coming mobile node.

\subsection{Mobility parameters' Service Level Specification}

In addition to the current service level specification (SLS) parameters that are more related to the QoS, we propose to consider those mobility parameters:

- Type_of_handover: layer 3, fast predictif, fast layer 2, smooth, seamless.

- Handover packet loss: $\min <\mathrm{HPL}<\max$

- Handover delay: $\min <\mathrm{HD}<\max$

Those mobility parameters can be used during the service negotiation in order to choose a fast, a smooth or a seamless handover. Based on the class of service, the network provider can specify the accepted values of the handover packet loss and the handover delay. He can also specify the best handover to be applied to support the class of service negotiated by the mobile node. Note that Mobile nodes and access routers can support one handover type or all of them. Before starting the handover procedure, the mobile node and the access router need to specify which the supported handover types are.

\subsubsection{Class of service mapping to the handover types}

During the handover, packets can be lost depending on the handover delay. For example we can use the mapping of the DiffServ services class as shown on table 1 . 


\begin{tabular}{|l|l|l|l|}
\hline QoS SLS & EF & AF & BE \\
\hline Mobility SLS & $-\quad \begin{array}{l}\text { Seamless handover } \\
\text { (fast and smooth): } \\
-\quad \text { - minimum handover } \\
\end{array}$ & $\begin{array}{l}\text { Fast or smooth } \\
\text { handover }\end{array}$ & $\begin{array}{l}\text { Standard mobility } \\
\text { management }\end{array}$ \\
& $-\begin{array}{l}\text { - minimum packet } \\
\text { loss }\end{array}$ & & \\
\hline
\end{tabular}

Table 1: Diffserv classes mapping to handover types.

The fast handover is achieved by the predictive handover supported by the PMM. The smooth handover is achieved by the packet forwarding mechanism from he cAR to nAR supported by the PM.

\subsubsection{Class of service Handover negotiation}

We propose to gather the set of access routers providing the same kind of handover as a domain with a policy mobility manager. And each time the mobile node arrives in a new domain, it will present only for the policy mobility manager which are the handovers it can support and not for each access router as in fast MIPV6. In fact, MIPv6 fast handover scheme suggests before starting the handover procedure to make the mobile node present to the access router which kind of handover it may support. The mobile node PMC will also negotiate with the PMM the handover parameters that it wishes to have in the domain. For this purpose the mobile node need only to provide its QoS SLS and the Policy Mobility Manager will achieve the mapping with the corresponding handover parameters.

\section{TESTBED IMPLEMENTATION AND FUTURE WORK}

This section describes the prototype implementation reported on Figure 7. The testbed is composed of 6 Personal Computers running the Linux RED Hat operating system and two laptops. The PCs are equipped with 100 $\mathrm{Mb} / \mathrm{s}$ ethernet cards. The core network is represented by the central PC, The two adjacent domains are the home and the foreign networks. Each domain contains a PMM on a PC configured as an Edge Router. A Base station connected to a $\mathrm{PC}$ configured as an access router where a policy mobility client (PMC) is deployed.

We used MIPv6 fast handover scheme for mobility management, and a Common Open Policy Service protocol (COPS) to transport the handover request and selection decisions from the PMM or the PDP to the PMC or the 
PEP. In the first step we deploy the PMC or PEP on the access router using COPS protocol, we think about deploying it in the mobile node as well.

At the PMM, we set up a mapping table between the Diffsev classes of service and the handover types.

When a mobile node arrives to the domain, it negotiates the handover corresponding to his class of service. We use two laptops, one with Fast Mobile IP (FMIP) protocol and one with FMIP protocol and PEP using COPS protocol. We consider the following scenarios :

- the PMM triggers the handover and interacts with the PMC to select the best cell to the mobile node.

- the MN triggers the handover using the mobility management protocol and the PMC on the access router interacts with the PMM to select the best cell.

- the PMC on the mobile node triggers the handover and interacts with the PDP to select the best cell

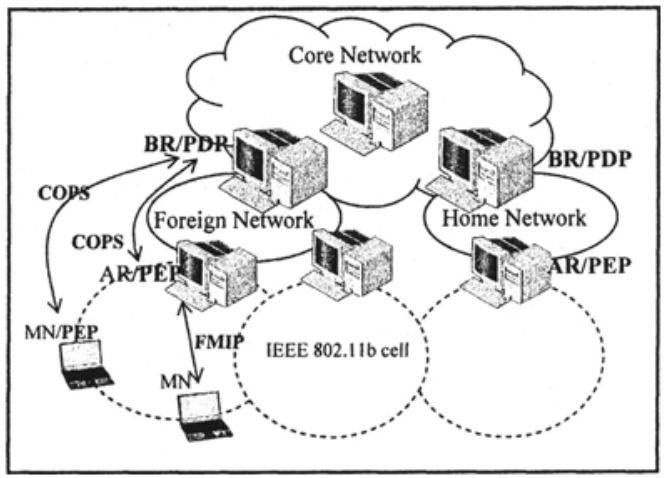

Figure $7:$ Testbed implementation

The modules that have been developed are shown on Figure 8. The COPS client side (PMC) interacts with the FMIPv6 or with a test application that can generate handover parameters requests. In the current version of the implementation the server is configured by reading a static table, where a mapping between the class of services and the handover parameters and type is provided. This means that the Decision module and the resource \& Topology model are too simplistic. We are working on the second version of the server, which should include topological information on wireless access networks, in order to better select the next cell of the mobile node. 


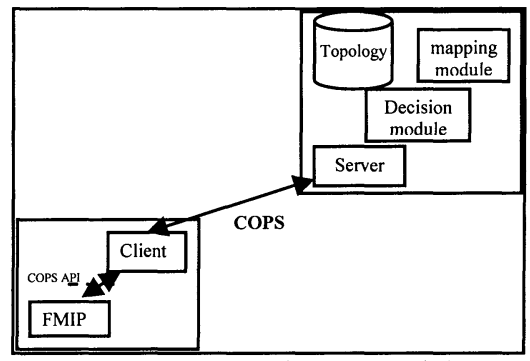

Figure 8 : PMM and PMC modules.

Work is also ongoing to measure the performance of the proposed solution in each scenario, aiming to infer some conclusions about the achieved handover delay and packet loss parameters.

\section{CONCLUSIONS}

In this article we have introduced a policy based handover concept which is a handover triggered not only on the fading signal strength but also on other constraints such as cost, resource availability or load balancing. We propose to combine the policy based management with a mobility management in order to support a predictive fast and smooth handover. We propose mobility parameters to be considered in a service level specification (SLS) and a mapping between the service classes and the handover types and parameters.

The policy mobility manager of the policy based architecture is responsible to trigger the policy based handover, to select the best next cell to the mobile node, to configure the current access router to forward mobile node packets and configure the context transfer to the new selected access router by interacting with the policy mobility client to enforce the handover and packet forwarding and context transfer decisions policies.

We finally present our testbed implementation where we deploy fast mobile IP to support the predictive fast and smooth handover and COPS to transport the policies between the PMM (PDP) and the PMC (PEP). Our ongoing work intends to measure the performance of this combined architecture regarding the delay handover and handover packet loss parameters. We also intend to finalise the policy information model necessary to support the proposed policy based handover management and support horizontal and vertical handovers. 


\section{REFERENCES}

[1] D. C Verna, Policy Based Networking: Architecture and Algorithms, New riders, 2002.

[2] D.Durham et al, "The COPS (Common Open Policy Service) Protocol", Internet RFC, Standard track, RFC 2748

[3] K.Chan et al "COPS Usage for Policy Provisioning (COPS-PR)," Internet RFC, Standard track, RFC 3084

[4] C. Perkins,. "Mobile IP", IEEE Communications

Magazine 35,5 (May 1997) pp. 84-99.

[5] A. Campbell et al, "Comparison of IP Micro-Mobility Protocols," IEEE Wireless Communications Magazine, Vol. 9, No. 1, February 2002.

[6] Ted Taekyoung Kwon et al, "Mobility Management for VoIP Service: Mobile IP vs. SIP," IEEE wireless communication magazine, Vol. 9, no.5, October 2002, pp.66-11

[7] A. T. Campbell et al, "Experiences with Cellular IP", Journal of High Speed Networks (JHSN), Special Issue on Multimedia in Wired and Wireless Environment, invited paper, September 2002.

[8] G. Dommety et al, "Fast Handovers for Mobile IPv6 ", Internet draft, 05 Mar 2003, draft-ietf-mobileip-fast-mipv6-06.txt

[9] M.O'Droma et al, "Always Best Connected" Enabled 4G Wireless World, IST Mobile and Wireless Communications Summit 2003, Aveiro, Portugal, June 15-18 2003.

[10] H. Chaouchi et al, "A Trial towards Unifying Control Protocols: COPS versus RADIUS/DIAMETER," IEEE conference MWCN, Stockholm

- September, 2002 